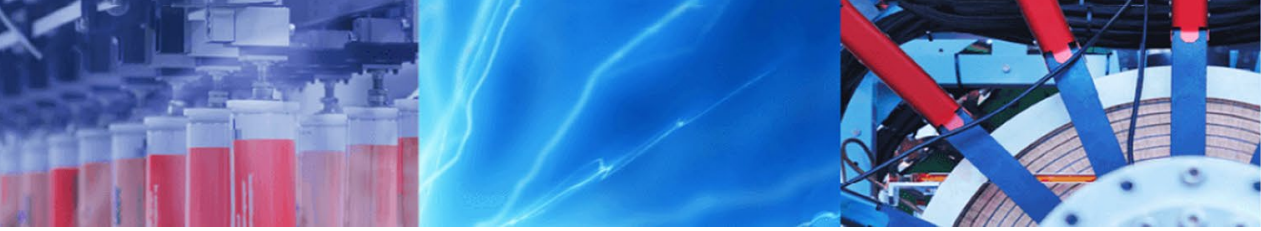

Research Article

\title{
Determination of the transverse modulus of cylindrical samples by compression between two parallel flat plates
}

\author{
Linda K. Hillbrick ${ }^{1}$ (D) Jamieson Kaiser ${ }^{1} \cdot$ Mickey G. Huson ${ }^{1} \cdot$ Geoffrey R. S. Naylor ${ }^{1}$ - Elliott S. Wise ${ }^{2}$. \\ Anthony D. Miller ${ }^{3}$. Stuart Lucas ${ }^{1}$
}

(c) Springer Nature Switzerland AG 2019

\begin{abstract}
Over the last century, several different theoretical models have been proposed for the calculation of the transverse modulus of fibres or cylinders from compression experiments. Whilst they all give similar results, the differences are significant enough to cause errors in computer simulation predictions of composite properties, and hence the issue warrants further investigation. Two independent approaches were applied to clarify this. Firstly, using an experimental approach, compression tests have been carried out on model elastic cylinders of poly(methyl methacrylate) as well as cuboids machined from the cylinders. The transverse modulus of this hard elastic material was determined directly from compression experiments on the cuboids and by analysis using different models for the cylinder compression data. Since machining was shown to change the modulus by virtue of relieving stresses in the samples, comparison was made between cuboids and machined cylinders. The transverse modulus obtained by direct compression of the cuboids was statistically equivalent to that obtained from the cylinders using the Morris model and was within $8 \%$ of the value obtained using the model derived by Jawad and Ward, as well as the mathematically equivalent models derived by Phoenix and Skelton and Lundberg. Finally, the separate and independent approach of finite element numerical modelling was also utilised. The finite element approach gave results that lie between the Jawad and Ward and Morris models. The close agreement in the outcomes of the finite element modelling and the experimental approach leads to the conclusion that the most accurate of the different analytical models are the equations by Morris as well as those due to Jawad and Ward, Phoenix and Skelton and Lundberg.
\end{abstract}

Keywords Transverse modulus · Fibres · Elastic cylinders · Compression · Finite element analysis · PMMA

\section{Introduction}

In recent times, there has been a significant increase in the use of fibre-reinforced polymer composites in a broad range of industries, including aerospace, military, engineering and sporting equipment. These materials offer significant advantages, mainly because of their lightweight, high strength, high modulus, high heat tolerance and chemical resistance properties.
Typically, high-performance fibres such as carbon, glass, Kevlar, UHMWPE and Vectran are used in a matrix of epoxy resin. The high strength and stiffness of these fibres comes from the highly oriented structure along the fibre axis. A consequence of this orientation is that fibres exhibit considerable anisotropy, where the mechanical properties in the longitudinal (axial) direction are different from those in the transverse direction [1-5]. The fibres are considered to be transversely isotropic because their properties do

$\triangle$ Linda K. Hillbrick, linda.hillbrick@csiro.au; Jamieson Kaiser, jamieson_73@hotmail.com; Mickey G. Huson, mickeyhuson@gmail.com; Geoffrey R. S. Naylor, drgeoffnaylor@gmail.com; Elliott S. Wise, ell.wise@gmail.com; Anthony D. Miller, tony.miller@bigpond.com; Stuart Lucas, stuart.lucas@csiro.au | ${ }^{1}$ CSIRO Manufacturing, Pigdons Road, Waurn Ponds, VIC 3216, Australia. ${ }^{2}$ CSIRO Computational and Simulation Science, Bayview Avenue, Clayton, VIC 3168, Australia. ${ }^{3}$ CSIRO Computational and Simulation Science, Waite Rd, Urrbrae, SA 5064, Australia. 
not differ significantly in directions perpendicular to the fibre axis [1].

In order to maximise the usage of this class of materials, it has become common to use computer simulation techniques to predict the elastic behaviour of fibre composites in service [6-9]. These simulations offer the opportunity to investigate a large range of parameters, which are often very difficult to access through experimental studies. The inputs required to model the material properties of a composite are accurate knowledge of the physical properties (elastic moduli, strength, conductivity, etc.) as well as the volume fractions of the individual components. Where the properties are anisotropic, they need to be known in all relevant directions. The material properties are generally well known or easily measured for the matrix material, which can be moulded into a test piece of suitable size and shape. The longitudinal properties of the reinforcing fibre are easily measured too; however, the transverse properties of the reinforcing fibre are considerably more difficult to determine. The internal structure and physical properties of reinforcing fibres are highly anisotropic, resulting in transverse mechanical properties which are vastly different from the corresponding longitudinal mechanical properties. Thus, for high-performance applications such as in aerospace, the lack of accurate information on the reinforcing fibres' transverse mechanical properties can be a limiting factor in modelling the critical performance characteristics of the manufactured fibre-reinforced composite.

The single fibre transverse compression test, where a cylindrical fibre is compressed between a pair of plane parallel plates (Fig. 1), is a widely reported method for determining the transverse compression properties of highly oriented fibres. As visible in Fig. 1, in this orientation, the force does not act on a sample of constant width and so it is not straight forward to extract the transverse modulus from the slope of the force-distance relationship. Indeed, there is no reason to even suspect the force-distance relationship to be linear. The method was first demonstrated for fibres by Hadley et al. [10], who used experimental load-displacement measurements to determine the transverse elastic moduli of poly(ethylene terephthalate), polyamide and polypropylene monofilaments. This technique has since been applied to study the transverse compression properties of numerous high-performance fibres including carbon fibre [2, 11, 12], graphite [13], Kevlar and other aramids [1-3, 13-16], polyesters [13, 17-19], polyamides $[13,14,17,19,20]$, polypropylene [17] and ceramic fibres $[2,14]$.

Early work $[10,17,19,21]$ focussed on the measurement of the contact width between the fibre and the compressing body. These fibres were generally thick monofilaments which allowed the contact area to be readily measured as

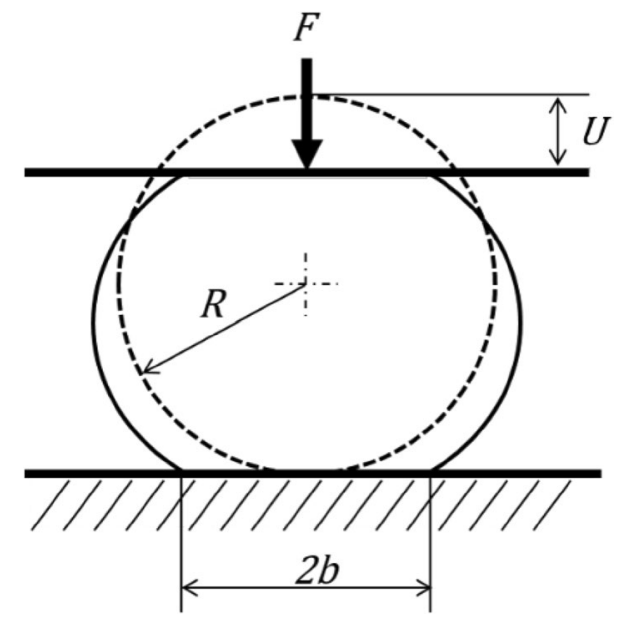

Fig. 1 Schematic diagram showing the compression of a cylinder of radius $\mathrm{R}$ between two parallel plates. Also shown are the force per unit length $(F)$, the contact width $(2 b)$ and the diametric compression $(U)$

a function of load as the fibres were compressed between transparent glass plates mounted on a microscope stage. In addition to measuring the contact width during compression of monofilaments, Pinnock et al. [19] and Hadley et al. [17] extended the investigation to include measurements of the expansion in the horizontal diametric plane. An expression for the change in diameter parallel to the plane of contact was derived in terms of the compliance constants and the applied load.

Whilst these methods were sufficiently accurate for relatively large fibres, finer fibres such as carbon $(<8 \mu \mathrm{m}$ diameter) required a different approach, viz. measuring the relative displacement of the loading plates. Over the course of 100 years, several researchers derived equations for this displacement in terms of the applied load and the elastic constants of the fibres [1, 13,22-26] (Table 1). Of all these equations, the Jawad and Ward equation [23] appears to have gained favour in textile research, being used by several workers $[2,11,12,14-16,18,27]$ in the last two decades to extract stiffness and other elastic parameters from experimental data. However, all of these equations involve approximations of some kind and there has been little attempt to test and evaluate the validity of the separate models. Whilst they all give similar results the differences are significant enough to cause errors in computer simulation predictions of composite properties and hence the issue warrants further investigation.

This study uses two independent approaches to compare these different equations. Firstly, macro-compression experiments have been carried out on model elastic cylinders of poly(methyl methacrylate) (PMMA) and the data analysed using all the models listed in Table 1. The results are compared to the transverse modulus determined 
directly on cuboids of PMMA machined from the cylinders. Preliminary results using this approach $[28,29]$ were inconclusive in delivering definitive support for one particular model. Subsequently, it has been identified that the technique used to correct for instrument compliance in these early experiments [28] may have been inadequate, particularly for hard samples. This has been addressed by using a video extensometer. The issue of the precision of compression testing as well as the potential effect on properties of the cylinders as a result of stress relief during machining are also now addressed.

In parallel with this experimental approach, threedimensional finite element modelling has also been used to determine the transverse modulus. This engineering approach is commonly adopted for complex-shaped samples and in this instance has the advantage of being valid for fibres with any theoretically possible elastic parameters. The results from the finite element approach are applied to the specific example of a PMMA cylinder and thereby test the various analytical models.

\section{Mathematical models}

The theoretical foundations of this method stem from the classical solution for the contact stresses between two smooth isotropic elastic bodies, first reported in Hertz's original papers in 1881 and 1882 and later reproduced and translated in his book [30, 31]. M'Ewen [21] extended Hertz's contact solution to account for the stresses between two elastic cylinders in contact. He considered the problem to be that of two semi-infinite bodies in contact along a very long narrow strip under the condition of plane strain and showed that if the contacting cylinders had similar elastic properties, then the contact half width (b) could be expressed in terms of the Young's modulus $(E)$, the load per unit length $(F)$, the radius of curvature $(R)$ of the contacting cylinders and Poisson's ratio $(v)$.

With an interest in fibres, Hadley et al. [10] further extended this work to account for the compression of anisotropic monofilaments between two rigid, parallel plates (Fig. 1). The anisotropic fibres possess transverse isotropy, the fibre axis being the axis of transverse isotropy. The contact half width $(b)$ was expressed in terms of the material constants as shown in Eq. 1

$b=\sqrt{\frac{4 F R}{\pi}\left(\frac{1}{E_{\mathrm{T}}}-\frac{v_{\mathrm{L}}^{2}}{E_{\mathrm{L}}}\right)}$

where the subscripts $T$ and $L$ indicate the transverse and longitudinal direction, respectively.

As stated earlier, contact width could not be measured with sufficient accuracy for finer fibres, necessitating a different approach. Morris [22] built on M'Ewan's work and derived an equation for the relative displacement of the plates $(U)$ in terms of the elastic constants of the fibre and the applied load per unit length $(F)$ (Table 1). This was followed by similar models derived by Phoenix and Skelton [13], Jawad and Ward [23] and more recently by Cheng et al. [1] (Table 1). Independent of the work on fibres, Sherif derived a similar equation for the compression of cylindrical carrots [26] and much earlier Foppl [24] and Lundberg [25] derived equations for the compression of cylindrical rollers and bearings. McCallion and Truong [32] later derived a more general equation for the compression of cylindrical rollers that reduced to either Foppl's or Lundberg's equation depending on the assumed pressure distribution. Other researchers [33-35] working on the compression of cylindrical rollers introduce an additional term to account for the contact deformation or deformation of

Table 1 Analytical formulae relating the transverse compression $(U)$ of anisotropic cylinders to the applied force per unit length $(F)$

\begin{tabular}{|c|c|c|c|}
\hline Year & Equation & Ref. & Eqn. \# \\
\hline 1907 & $U=\frac{4 F}{\pi}\left(\frac{1}{E_{T}}-\frac{v_{\mathrm{L}}^{2}}{E_{\mathrm{L}}}\right)\left(\operatorname{Ln}\left(\frac{2 R}{b}\right)+\frac{1}{3}\right)$ & Foppl [24] & 2 \\
\hline 1949 & $U=\frac{4 F}{\pi}\left(\frac{1}{E_{\mathrm{T}}}-\frac{v_{\mathrm{L}}^{2}}{E_{\mathrm{L}}}\right)\left(\operatorname{Ln}\left(\frac{4 R}{b}\right)-\frac{1}{2}\right)$ & Lundberg [25] & 3 \\
\hline 1968 & $U=\frac{4 F}{\pi}\left(\frac{1}{E_{\mathrm{T}}}-\frac{v_{\mathrm{L}}^{2}}{E_{\mathrm{L}}}\right)\left(\sinh ^{-1}\left(\frac{R}{b}\right)\right)$ & Morris [22] & 4 \\
\hline 1974 & $U=\frac{4 F}{\pi}\left(\frac{1}{E_{\mathrm{T}}}-\frac{v_{\mathrm{L}}^{2}}{E_{\mathrm{L}}}\right)\left(\sinh ^{-1}\left(\frac{2 R}{b}\right)-\frac{1}{2}\right)$ & Phoenix and Skelton [13] & 5 \\
\hline 1976 & $U=\frac{4 F}{\pi}\left(\frac{1}{E_{\mathrm{T}}}-\frac{v_{\mathrm{L}}^{2}}{E_{\mathrm{L}}}\right)\left(\operatorname{Ln}\left(\frac{2 R}{b}\right)+\frac{1}{2}\right)$ & Sherif et al. [26] & 6 \\
\hline 1978 & $U=\frac{4 F}{\pi}\left(\frac{1}{E_{\mathrm{T}}}-\frac{v_{\mathrm{L}}^{2}}{E_{\mathrm{L}}}\right)\left(\sinh ^{-1}\left(\frac{R}{b}\right)+0.19\right)$ & Jawad and Ward [23] & 7 \\
\hline 2004 & $U=\frac{4 F}{\pi b^{2}}\left[\left(-\frac{v_{\mathrm{T}}}{E_{\mathrm{T}}}-\frac{v_{\mathrm{L}}^{2}}{E_{\mathrm{L}}}\right)\left(\sqrt{b^{2}+R^{2}}-R\right) R+\left(\frac{1}{E_{\mathrm{T}}}-\frac{v_{\mathrm{L}}^{2}}{E_{\mathrm{L}}}\right) b^{2} \operatorname{Ln} \frac{\sqrt{b^{2}+R^{2}}+R}{b}\right]$ & Cheng et al. [1] & 8 \\
\hline
\end{tabular}


the plate. We have ignored these latter works because for the compression of fibres the plate is generally much stiffer than the fibre, and hence deformation of the plate is expected to be minimal. The equations of interest in the compression of fibres between two parallel plates are shown in Table 1.

Which of these models is the most accurate? When the equations are plotted, we note that Eqs. 3, 5 and 7 are indistinguishable from each other (Fig. 2). This is not surprising if we note that quite generally

$\sinh ^{-1}\left(\frac{R}{b}\right)=\operatorname{Ln} \frac{\sqrt{b^{2}+R^{2}}+R}{b}$

and so for $R \gg b$

$\operatorname{Ln}\left(\frac{2 R}{b}\right) \approx \sinh ^{-1}\left(\frac{R}{b}\right)$

and thus Eqs. (5) and (7) both transform to:

$U=\frac{4 F}{\pi}\left(\frac{1}{E_{\mathrm{T}}}-\frac{v_{\mathrm{L}}^{2}}{E_{\mathrm{L}}}\right)\left(\operatorname{Ln}\left(\frac{4 R}{b}\right)-\frac{1}{2}\right)$

which is the equation derived by Lundberg in 1949 .

On the other hand, the models due to Foppl and Sherif et al. predict fibre properties that are slightly less stiff and Morris's, as well as Cheng's model, predict stiffer fibres (Fig. 2).

Phoenix and Skelton [13] questioned the manner in which Morris [22] superimposed the stress fields as well as his neglect of certain terms in the derivation and some of his approximations. Jawad and Ward point out that their result is very similar to that previously obtained by Foppl [24],

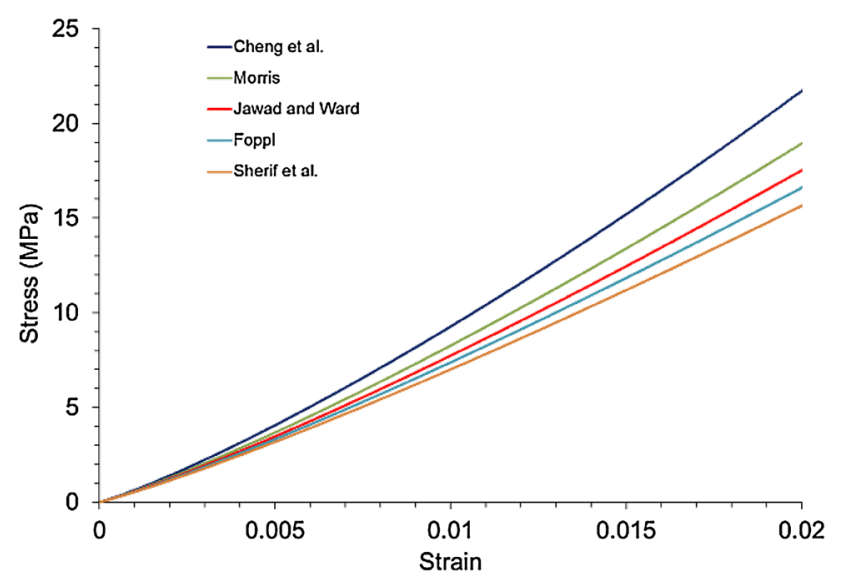

Fig. 2 Comparison of the different models for the compression of a cylinder of constant radius $(12.5 \mathrm{~mm})$, plotted with values of the elastic parameters: $E_{\mathrm{T}}(3000 \mathrm{MPa}), E_{\mathrm{L}}(4000 \mathrm{MPa}), v_{\mathrm{L}}(0.399)$ and $v_{\mathrm{T}}$ (0.399). The plot for the models proposed by Phoenix et al. and Lundberg are indistinguishable from that due to Jawad and Ward the difference being due to the fact that Foppl assumed a parabolic distribution of stress in the contact zone, whereas Jawad and Ward followed Hertz and assumed an elliptical stress distribution. McCallion and Truong [32] derived a more general relationship (Eq. 12) for the compression of a cylinder, which included a term $\beta$ which described the pressure distribution across the contact:

$U=\frac{4 F}{\pi}\left(\frac{1}{E_{\mathrm{T}}}-\frac{v_{\mathrm{L}}^{2}}{E_{\mathrm{L}}}\right)\left(\operatorname{Ln}\left(\frac{2 R}{b}\right)-1+0.5[\Psi(\beta+1.5)-\Psi(0.5)]\right)$

where $\Psi(z)=\mathrm{d}(\operatorname{Ln}[\Gamma(z)]) / \mathrm{d} z$ and $z$ is the vertical distance, along the diameter, from the surface of the cylinder and $\Gamma(z)$ is the Gamma function.

For the special case of $\beta=0.5$ which describes an elliptical pressure distribution, the equation reduces to Lundberg's equation and for $\beta=1$ which describes a parabolic pressure distribution, the equation reduces to that due to Foppl.

All of the analytical formulae in Table 1 make a number of assumptions in their derivations. These include the system being in a state of plane strain and the applied force taking on a particular distribution over the contact width. Equations (2)-(7) additionally make the assumption that the contact width is small compared with the fibre's radius $(b \ll R)$ resulting in formulae that only depend on the elastic parameters $E_{T}, E_{\mathrm{L}}$ and $v_{\mathrm{L}}$ and do not include the additional elastic constant $v_{\mathrm{T}}$, the transverse Poisson's ratio. Note too that whilst the equations in Table 1 all contain the half contact width $(b)$ it is not necessary to measure $b$ directly as it is a function of $F, E_{T}, E_{\mathrm{L}}$ and $v_{\mathrm{L}}$ (Eq. 1). Equation 1 can be substituted into Eqs. 2-8, and, provided $E_{\mathrm{L}}$ and $v_{\mathrm{L}}$ are known, $E_{\mathrm{T}}$ can be calculated directly from the force per unit length $(F)$ versus diametric compressive displacement (U) data (Fig. 2).

\section{Experimental and finite element modelling methods}

\subsection{Materials}

Poly(methyl methacrylate) (PMMA) was selected as representative of a hard elastic material. Cylindrical rods of the material (EFM Plastics), with a diameter of $25 \mathrm{~mm}$, were cut into lengths of $50 \mathrm{~mm}$. After testing, some of the cylinders were machined into new cylinders with a diameter of $20 \mathrm{~mm}$ and some were machined into cuboids with sides of $15 \mathrm{~mm}$.

\subsection{Compression}

\subsubsection{General}

Compression was carried out on an Instron tester (model 5967) fitted with an advanced non-contacting 
video extensometer (model 2663-821) to avoid instrument compliance, which was a significant factor for the compression of the stiff PMMA samples. Direct measurement of the sample strain overcomes the problem of instrument compliance. Samples were compressed both axially and transversely at a rate of $25 \% / \mathrm{min}$ to a strain level of $2-5 \%$, and data were collected using Bluehill 3 software. The reflective spots used by the video extensometer were placed on metal plates above and below the samples so that the effective gauge length of the sample was the cylinder diameter, cylinder length, cuboid length or cuboid width (Fig. 3). Care was taken to adjust the platens parallel to one another by bringing them together under a load of $500 \mathrm{~N}$ before final tightening of the locking nuts. Once the Instron was set up, all tests were done with no further adjustment in order to minimise any effects due to instrument setup.

\subsubsection{Poisson's ratio}

For the compression experiments to measure Poisson's ratio, two sets of reflective spots were placed on the cuboid samples such that both the axial and the transverse strain could be measured (Fig. 3c). The Poisson's ratio was obtained by measuring both the axial compression and the transverse expansion of a cuboid during an axial compression test. The slope of the strain-force curve was measured between 2000 and $12,000 \mathrm{~N}$, and Poisson's ratio was calculated [36] from:

$\nu_{\mathrm{L}}=\frac{\mathrm{d} \varepsilon_{\text {transverse }} / \mathrm{d} F}{\mathrm{~d} \varepsilon_{\text {axial }} / \mathrm{d} F}$

The average of 10 independent tests on each of two of the PMMA cuboids is reported.

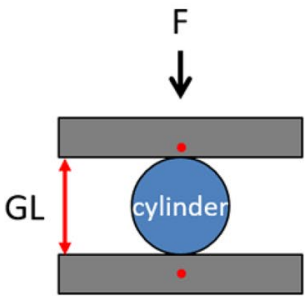

(a)

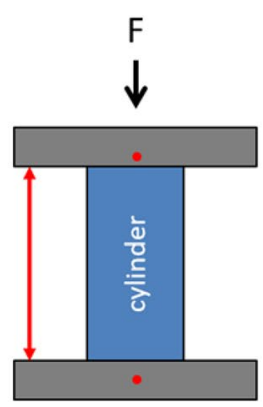

(b)
Fig. 3 Experimental setup for compression of cylinders and cuboids between parallel plates and under an applied force $(F)$. a cylinder compressed in the transverse direction, b cylinder compressed in the axial direction, c cuboid compressed in the axial direction and $\mathbf{d}$ cuboid compressed in the transverse direction.

\subsubsection{Axial compression}

Ten axial force compression experiments were performed on each of ten original cylinders, five machined cylinders and five cuboid samples. Care was taken to position the samples centrally on the bottom plate (on the axis of the load cell) before each test. The moduli were determined from the linear slope of the stress strain curves in the region from 10 to $30 \mathrm{MPa}$.

\subsubsection{Transverse compression}

Transverse force compression experiments were performed 10 times in both orthogonal directions on each of the cuboids. The moduli of the cuboids in the transverse direction were determined from the linear slope of the stress strain curves in the region from 10 to $20 \mathrm{MPa}$.

Transverse moduli for the cylinders were obtained from the transverse force compression data by fitting the equations in Table 1 to the data using the experimentally determined values of $E_{\mathrm{L}}$ (Table 3 ) and $v_{\mathrm{L}}$. For the Cheng equation, $v_{T}$ was assumed to be equal to $v_{\mathrm{L}}$. Note that the modulus is not very sensitive to $v_{T}$; a $10 \%$ change results in less than a $1 \%$ change in modulus. The raw data contain data prior to contact and due to the imperfect alignment of the compression plates, the data in the initial part of the force compression curve may be unreliable [2]. Furthermore, there is considerable uncertainty in the displacement value at the zero load position $[2,18]$; thus, a method is needed to reliably pick the contact point. Similar to the method used by Kotani et al. [18], we included an offset value $\Delta U$ in the equations shown in Table 1 in order to take into account both the zero load data and the uncertainty in the contact point. This allowed the data to be fitted simultaneously for both the transverse modulus and the offset $(\Delta U)$ using non-linear least squares routines

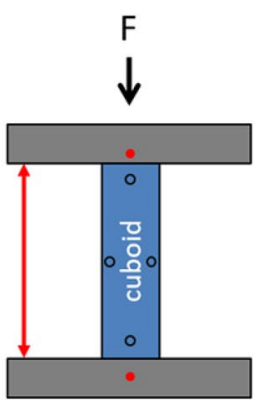

(c)

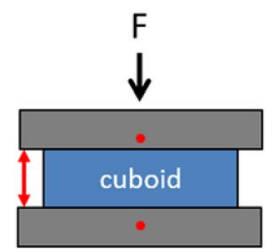

(d)
Solid red circles show the position of the reflective spots for general compression, and arrows indicate the effective gauge length (GL). Hollow circles on the cuboid setup for axial compression show the position of the reflective spots for the Poisson's ratio experiment 
written in Matlab (MathWorks, Massachusetts, USA). The data have been fitted in the region from 0.5 to $1.5 \%$ strain. This avoids the imprecise initial data and the non-elastic behaviour at higher strains. Kar et al. [27] compressed composite rods and showed that the Jawad and Ward equation gave a steady state value of $E_{\mathrm{T}}$ between 0.4 and $0.7 \%$ strain. Ten transverse force compression experiments were performed on each of the ten original cylinders and five machined PMMA cylinders.

\subsection{Finite element modelling}

Finite element techniques were adopted to model the transverse compression of a cylindrical body. Using this approach, the full elastic parameter space for a transversely isotropic material was explored. Noting the bounds on the elastic parameter space for isotropic materials $(E>0$, and $-1 \leq v \leq 0.5)$ and in the transverse isotropic case $\left(E_{\mathrm{T}}, E_{\mathrm{L}}>0\right.$, and $-1<v_{\mathrm{T}}<1-2 v_{\mathrm{L}}^{2} / E_{\text {rat }}$, where $E_{\mathrm{rat}}=E_{\mathrm{L}} / E_{\mathrm{T}}$ is a measure of the fibre's degree of anisotropy) [37], it was decided to uniformly sample $1 / E_{\mathrm{rat}}, v_{\mathrm{T}}$ and $v_{\mathrm{L}}$. To do this, samples were iteratively generated until the full theoretical parameter space had been explored. This required 1008 samples in total. A full 3D model was used thus avoiding the need to invoke the plane strain assumption. Small displacement elasticity, with no friction at the plate-cylinder interface, was used.

To compare the results of the finite element modelling with that of the analytical models, it is noted that the finite element modelling computes a force for a given displacement, whereas the analytical formulae perform the inverse operation. Hence, for comparison purposes, using a particular example the $3 \mathrm{D}$ finite element model was first used to compute a force at a $2 \%$ nominal strain, and then the analytical model used to invert this force. The absolute relative error was then computed between the original $2 \%$ strain and the inverted strain.

\section{Results and discussion}

\subsection{Instrumentation issues}

Preliminary work by Hillbrick et al. [28] used platen on platen compression curves to correct for compliance; however, it subsequently became apparent that this was inadequate and all further work used a video extensometer for greater accuracy of strain measurement. Setting the Instron up to do compression tests involves securing the bottom compression plate to the instrument frame and the top compression plate to the load cell. In both cases, the plates are screwed into position and secured with locking nuts. Tests to assess the precision of compression testing highlighted that during this assembly alignments can change, which in turn significantly affects the results. Clearly, for any comparisons to be made between transverse moduli of cylinders and cuboids, the Instron setup must remain unchanged. The use of a video extensometer and an instrument that remained unchanged throughout the experiment allowed any inaccuracies in measurement to be minimised.

\subsection{Poisson's ratio}

Axial compression of a cuboid whilst measuring the strain in both the axial and transverse direction yields the result shown in Fig. 4. The strain in both directions varies linearly with the application of increased load. Poisson's ratio, given by the ratio of the slope of the transverse curve to that of the axial curve, was shown to be $0.399 \pm 0.018$ standard deviation. This compares well with values in the literature of $0.40 \pm 0.02$ for injection moulded PMMA [36].

\subsection{Effect of machining}

Examination of the PMMA samples under crossed polars showed that residual stress was clearly present, as indicated by the coloured bands (stress decreases in the order blue, red, yellow, white and black) (Fig. 5). After machining, there is a reduction in stress levels for both the cylinders and the cuboids. The experimental design involves comparison of the transverse modulus of the PMMA, as measured directly by compression of a machined cuboid, with the results obtained by analysing the data from the compression of cylinders using the equations in Table 1. Thus, it is important to establish whether the removal of stress by machining results in a change in modulus of the

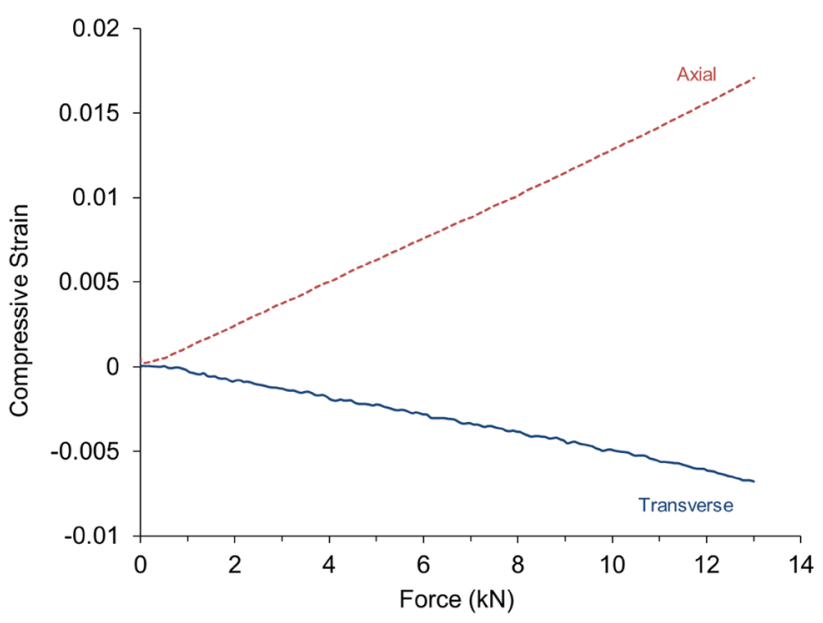

Fig. 4 Typical strain-force curves for the axial compression (dashed line) and transverse dilation (solid line) of a PMMA cuboid 


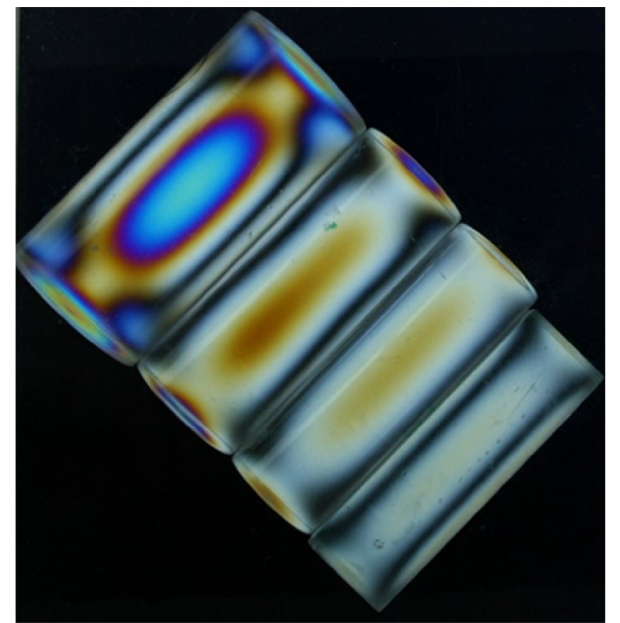

Fig. 5 Stress patterns in a PMMA cylinder and machined PMMA cylinders and a cuboid when viewed under crossed polars. Diagonally from the top left: unmachined cylinder, two machined cylinders and a machined cuboid

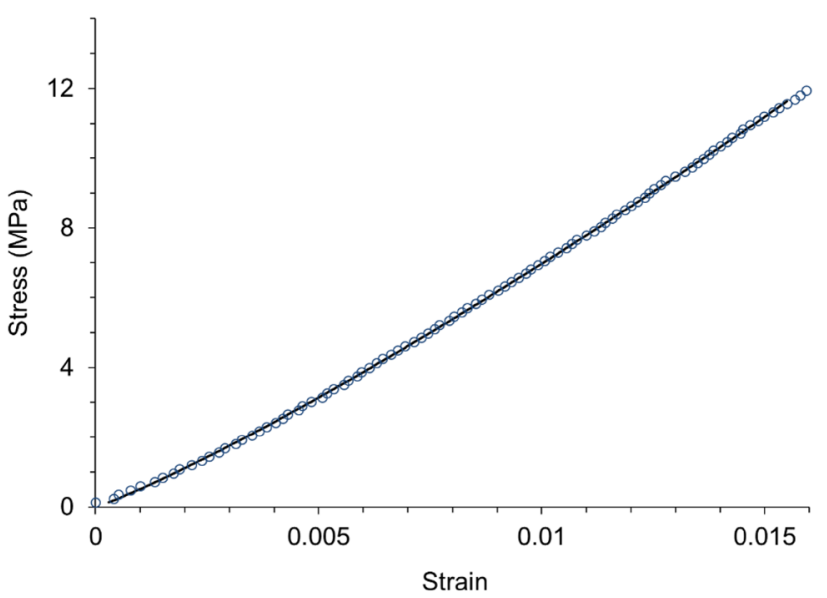

Fig. 6 Typical stress-strain curve for the transverse compression of a cylinder (blue open circles) as well as the best fit of the Jawad and Ward equation (solid black line)

material. To this end, five 25-mm-diameter cylinders were tested transversely and longitudinally (axially), machined to smaller diameter $(20 \mathrm{~mm})$ cylinders and then retested as before.

Load displacement curves from the transverse compression of PMMA cylinders were fitted using the Jawad and Ward equation in Table 1, fixing $E_{\mathrm{L}}$ and $v_{\mathrm{L}}$ and allowing $E_{\mathrm{T}}$ and the offset $\Delta U$ to vary. The data were well fitted with $R^{2}$ values $>0.99$, Fig. 6 showing a typical stress-strain curve for the transverse compression of a cylinder fitted with the Jawad and Ward equation (this equation was chosen initially because of the results presented in later sections).
Table 2 shows these results, as well as the results from testing an additional five cylinders and the cuboids machined from these cylinders. The transverse moduli of the original cylinders are reasonably consistent, with a coefficient of variation of less than $1 \%$ for the ten cylinders tested (Table 2). The precision of measuring the longitudinal moduli was slightly poorer with a coefficient of variation of less than $2 \%$. Note that the lower transverse modulus (2965 MPa) compared to the longitudinal modulus (3509 MPa) is consistent with some orientation along the length of the PMMA rod. As noted earlier, examination of the samples under crossed polars showed some birefringence, supporting the existence of orientation.

All five cylinders show a significant increase in both transverse and longitudinal modulus upon machining to a smaller diameter (Table 2), presumably as a result of the release of stress brought about by machining. The transverse modulus of the cylinders increased by $4.4 \%$ on machining, and, ignoring the anomalous longitudinal modulus for cylinder 5 , the longitudinal modulus has increased by about 7\%. Note that the equations in Table 1 are relatively insensitive to longitudinal modulus, and thus the result for the machined cylinder 5 does not lead to a transverse modulus that is an outlier. The result has, however, been disregarded when averages are calculated (Table 2). Comparable to the machined cylinders, the longitudinal modulus of the cuboids ( $3736 \mathrm{MPa}$ ) is slightly greater $(5 \%)$ than the cylinders from which they were machined (3549 MPa) (Table 2). Thus, machining leads to stress relaxation and an increase in modulus, requiring that in order to test the different models we need to compare the data from the cuboids with that from the machined cylinders. Encouragingly, the longitudinal modulus of the cuboids is only $0.4 \%$ larger than that of the machined cylinders (3721 MPa after removing the anomalous data point). This difference is not statistically significant. These results are consistent with those of researchers studying soda-lime glass [38], who observed that reducing the tensile stress in a sample led to an increased modulus.

\subsection{Comparison of models}

Table 2 shows the results for both the transverse and the longitudinal moduli of the cuboids machined from the cylinders. The transverse modulus of the five cuboids was $2867 \pm 164 \mathrm{MPa}$ and the longitudinal modulus $3736 \pm 26 \mathrm{MPa}$. A typical stress-displacement curve for the transverse compression of a PMMA cuboid is shown in Fig. 7.

Load displacement curves from the transverse compression of the machined cylinders were also fitted using the rest of the equations in Table 1 (all $R^{2}$ values $>0.99$ ). 
Table 2 Transverse $\left(E_{T}\right)$ and longitudinal $\left(E_{\mathrm{L}}\right)$ Young's modulus of original $(\phi=25 \mathrm{~mm})$ and machined $((\phi=20 \mathrm{~mm})$ cylinders as well as cuboids machined from cylinders 6-10

\begin{tabular}{|c|c|c|c|c|c|c|c|c|c|c|c|c|c|c|}
\hline \multirow[t]{2}{*}{ Cylinder } & \multicolumn{4}{|c|}{ Original cylinder ( $\phi=25 \mathrm{~mm})$} & \multicolumn{4}{|c|}{ Machined cylinder $(\phi=20 \mathrm{~mm})$} & \multicolumn{4}{|c|}{ Machined cuboid } & \multicolumn{2}{|c|}{$\begin{array}{l}\text { Difference } \\
\text { (\%) }\end{array}$} \\
\hline & $E_{\mathrm{T}}(\mathrm{MPa})$ & SD & $E_{\mathrm{L}}(\mathrm{MPa})$ & SD & $E_{\mathrm{T}}(\mathrm{MPa})$ & SD & $E_{\mathrm{L}}(\mathrm{MPa})$ & SD & $E_{\mathrm{T}}(\mathrm{MPa})$ & SD & $E_{\mathrm{L}}(\mathrm{MPa})$ & SD & $E_{\mathrm{T}}$ & $E_{\mathrm{L}}$ \\
\hline 1 & 2992 & 31 & 3459 & 76 & 3080 & 68 & 3693 & 35 & & & & & 3 & 7 \\
\hline 2 & 2951 & 51 & 3450 & 89 & 3083 & 68 & 3727 & 35 & & & & & 4 & 8 \\
\hline 3 & 2984 & 47 & 3473 & 45 & 3107 & 75 & 3724 & 37 & & & & & 4 & 7 \\
\hline 4 & 2951 & 48 & 3514 & 88 & 3158 & 22 & 3742 & 40 & & & & & 7 & 6 \\
\hline 5 & 2983 & 33 & 3445 & 68 & 3089 & 143 & 5240 & 152 & & & & & 4 & 52 \\
\hline 6 & 2946 & 49 & 3596 & 237 & & & & & 3135 & 320 & 3702 & 40 & 6 & 3 \\
\hline 7 & 2944 & 28 & 3554 & 73 & & & & & 2870 & 95 & 3763 & 39 & -3 & 6 \\
\hline 8 & 2956 & 21 & 3521 & 74 & & & & & 2849 & 134 & 3761 & 22 & -4 & 7 \\
\hline 9 & 2957 & 39 & 3519 & 53 & & & & & 2782 & 85 & 3723 & 59 & -6 & 6 \\
\hline 10 & 2984 & 29 & 3555 & 88 & & & & & 2700 & 247 & 3730 & 30 & -10 & 5 \\
\hline Average & 2965 & 38 & 3509 & 89 & 3103 & 75 & $3722^{*}$ & $37^{*}$ & 2867 & 176 & 3736 & 38 & & \\
\hline SD & 19 & & 51 & & 32 & & $21^{*}$ & & 164 & & 26 & & & \\
\hline CV (\%) & 0.6 & & 1.5 & & 1.0 & & $0.6^{*}$ & & 5.7 & & 0.7 & & & \\
\hline
\end{tabular}

Transverse modulus of cylinders determined using the Jawad and Ward equation

*Values from cylinder 5 omitted

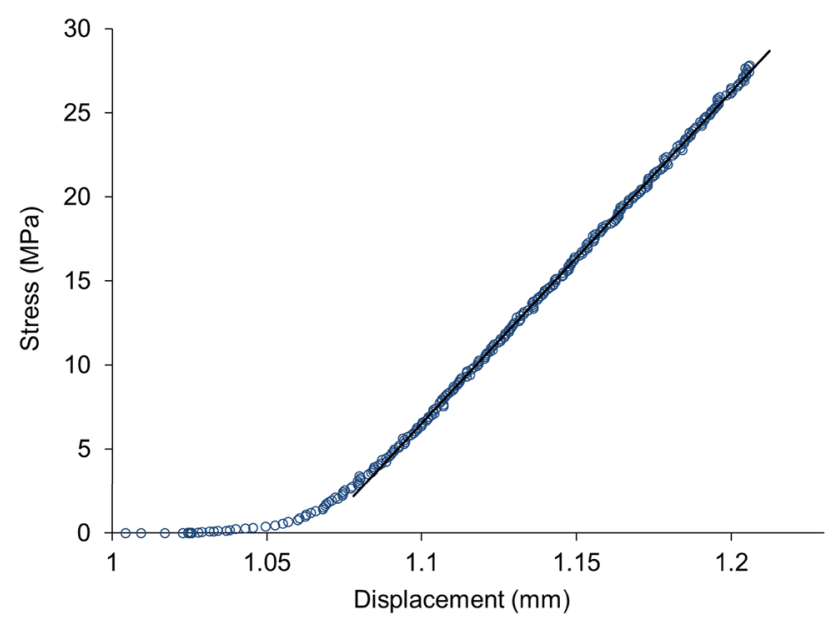

Fig. 7 Typical stress-displacement curve for the transverse compression of a PMMA cuboid. Also shown is the trendline of the linear region (10-20 MPa) used to determine the modulus
Since the release of stress brought about by machining results in an increase in modulus, the values obtained by the direct transverse compression of the cuboids need to be compared to the values for the 20-mm-diameter cylinders, obtained using the different models. When this is done (Table 3), the best match is the model due to Morris which gives a result that is within approximately $1 \%$ of the value obtained directly. A student $t$ test gives a $\mathrm{p}$ value of 0.654 showing that statistically there is no difference between the value obtained using Morris's equation and the value obtained directly by compression of the cuboids. The next best match is obtained when the model derived by Jawad and Ward is used, resulting in a transverse modulus $8 \%$ higher than that obtained directly. A student $t$ test shows that this difference is significant. Remember too that the models derived by Phoenix et al. and Lundberg are mathematically equivalent to that derived by Jawad and Ward, so these equations also predict a transverse modulus that is $8 \%$ too high. The Cheng equation predicts a modulus that is $10 \%$ too low whilst Foppl's and Sherif
Table 3 Transverse Young's modulus $\left(E_{\mathrm{T}}\right)$ of machined $(\phi=20 \mathrm{~mm})$ cylinders calculated using different models and compared to the transverse modulus of the cuboid $(2867 \pm 164 \mathrm{MPa})$

\begin{tabular}{llllllll}
\hline Model & $n$ & \multicolumn{2}{l}{ Diameter $20 \mathrm{~mm}$} & & $\begin{array}{l}\text { Difference from } \\
\text { cuboid (\%) }\end{array}$ & $p$ & Significance \\
\cline { 2 - 5 } & & $E_{\mathrm{T}}(\mathrm{MPa})$ & $\mathrm{SD}$ & & & \\
\hline Cheng & 5 & 2594 & 26 & -9.5 & 0.020 & $y$ \\
Morris & 5 & 2903 & 32 & 1.3 & 0.654 & $n$ \\
Jawad and Ward & 5 & 3103 & 32 & 8.2 & 0.031 & $y$ \\
Foppl & 5 & 3248 & 33 & 13.3 & 0.006 & $y$ \\
Sherif & 5 & 3415 & 34 & 19.1 & 0.001 & $y$ \\
\hline
\end{tabular}


et al.'s equations predict values that are too high by $13 \%$ and $19 \%$, respectively.

Sherif et al. [26] also compressed PMMA cylinders and compared the results obtained using their equation with that obtained using the equations of Foppl [24] and also Lundberg [25] (which they ascribe to Dorr [39]). Their results show the Sherif equation to be the best predictor, followed by that of Foppl and finally Lundberg. It should be noted, however, that Sherif et al. [26] do not mention compliance issues, nor instrument setup problems, which we found to be significant. Furthermore, they assumed the PMMA cylinders to be isotropic, measuring the modulus by an axial compression of the cylinders. PMMA cylinders are almost always anisotropic with $E_{\mathrm{L}}>E_{\mathrm{T}}$ (see Table 2). Using an experimental modulus that is too high will automatically favour the Sherif model which predicts the highest modulus (Table 3). The results from the preliminary work of Hillbrick et al. [28] favoured the Cheng et al. equation, however, this data were corrected for compliance using platen on platen compression curves which showed more than 300 micrometres of compliance at the loads needed to compress the PMMA cylinders. The compression of the PMMA cylinders was approximately 400 micrometres; hence, a correction that is almost as large as the actual measurement is unlikely to result in very accurate data. Later work using a video extensometer [29] recommended either the Jawad and Ward equation or the Foppl equation although in this work attention was not paid to the effects of instrument setup.

\subsection{Finite element modelling}

Figure 8 shows the absolute relative errors in displacement between the results obtained using the three-dimensional finite element modelling and that obtained using two of the analytical models, namely (a) Jawad and Ward and (b) Cheng et al. Recall that the finite element calculations relate to a nominal $2 \%$ state of strain.
Figure 8a illustrates that in the case of the Jawad and Ward model, the relative difference from the finite element modelling can be determined fully based on the non-dimensional fraction $v_{\mathrm{L}} / \sqrt{ } E_{\text {rat }}$. The differences are smallest when $v_{\mathrm{L}} / \sqrt{ } E_{\text {rat }}$ is close to zero and increase as $v_{\mathrm{L}} / \sqrt{ } E_{\text {rat }}$ increases or decreases. In the practical example of the PMMA cylinders used in the experimental section of the work, $v_{\mathrm{L}}, E_{\mathrm{L}}, E_{\mathrm{T}}$ were measured experimentally to be $0.399,3736 \mathrm{MPa}$ and $2867 \mathrm{MPa}$, respectively. This gives a value of 0.35 for the $x$ axis (i.e. $v_{\mathrm{L}} / \sqrt{ } E_{\text {rat }}$ ) in Fig. 8a. At a $2 \%$ nominal strain, the finite element model computes an absolute difference of $5 \%$ relative to the Jawad and Ward analytical model (Fig. 8a). Thus, for the same force, or stress, when the finite element model gives $2 \%$ strain the Jawad and Ward model will predict either $2.1 \%$ (5\% higher) or $1.9 \%$ (5\% lower). Considering the stresses at these strains, we note that the stress predicted by the finite element model, at $2 \%$ strain, will be $6 \%$ higher or lower than that predicted by the Jawad and Ward model.

Using the graphical representation of the different analytical models in Fig. 2, the two possible options for positioning of the finite element model would place it either slightly below the curve corresponding to the Morris equation (approximately $8 \%$ higher stress at $2 \%$ strain than Jawad and Ward) or close to the curve corresponding to the Foppl equation (5\% lower stress at $2 \%$ strain than Jawad and Ward).

Turning to the relationship between the absolute relative difference between the Cheng et al. model and the finite element modelling shown in Fig. 8b, the difference is now a function of both $v_{\mathrm{L}} / \sqrt{ } E_{\text {rat }}$ and $v_{\mathrm{T}}$, i.e. forming a surface in $v_{\mathrm{L}} / \sqrt{ } E_{\text {rat }}$ and $v_{T}$ space. This surface has been projected and averaged into the hexagon-bin plot in Fig. 8b. Compared to Fig. $8 \mathrm{a}$, it can be seen that in this case the relative errors follow a more complex pattern with the minimal difference (schematically shown as the hexagons shaded in dark blue in Fig. 8b) forming an approximate parabola passing through the points $( \pm 0.5,-1.0)$ and $(0.0,-0.5)$ in $\left(v_{\mathrm{L}} / \sqrt{ } E_{\text {rat },}, v_{\top}\right)$ space. Again, in the practical example of the
Fig. 8 Absolute errors relative to the 3D FEM for the Jawad and Ward (a) and Cheng et al. (b) analytical fibre models. Errors were computed at $2 \%$ nominal strain for 1008 uniformly distributed parameter space points choosing $E_{\mathrm{L}}=3.3 \mathrm{GPa}$ and $E_{\text {rat }} \in[1 ; 100)$. Relative errors are in displacements. Plots are coloured using the same scale to assist with comparison (a)

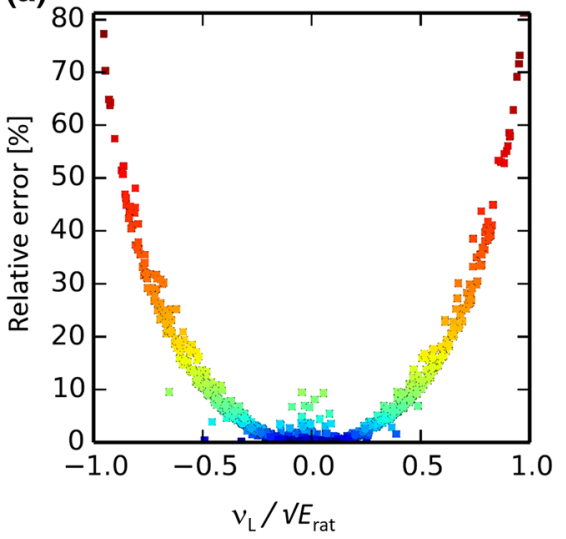

(b)

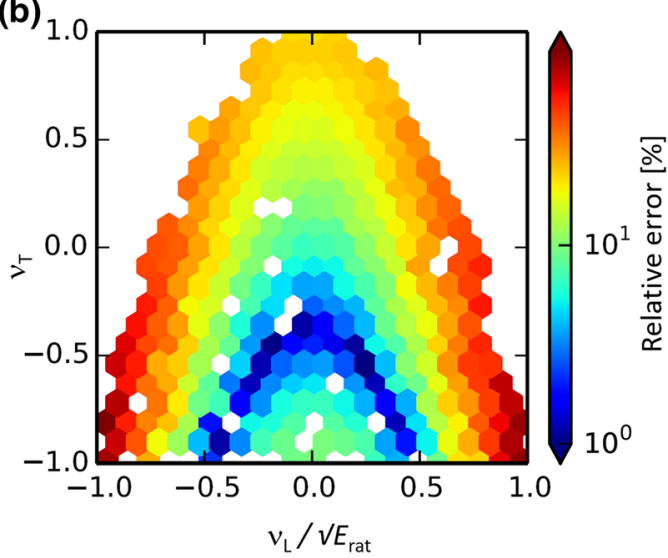


PMMA cylinders used in the experimental section of the work, the relevant value for the $x$ axis (i.e. $v_{\mathrm{L}} / \sqrt{E}_{\text {rat }}$ ) in Fig. $8 \mathrm{~b}$ is also 0.35 . The transverse Poisson's ratio, $v_{T}$, i.e. the value for the vertical axis in Fig. $8 \mathrm{~b}$ has not been independently determined. However, it is expected to be in the range $0.2-0.5[40,41]$ and a reasonable first-order approximation is that $v_{\mathrm{T}}=v_{\mathrm{L}} \approx 0.4$. Using this value, Fig. $8 \mathrm{~b}$ indicates that in this example the absolute relative difference between the strain computed by the Cheng et al. model and the finite element modelling is approximately $15 \%$. This translates to a finite element model curve that at $2 \%$ strain would have a stress either $3 \%$ or $48 \%$ above the Jawad and Ward equation.

Considering both sets of results, the most probable positioning of the finite element model curve is $3 \%$ above (from comparison with the Cheng et al. model) or $6 \%$ above (from comparison with the Jawad and Ward equation model) the Jawad and Ward equation. In other words, we must conclude that the finite element modelling result lies between the Jawad and Ward and Morris models. Encouragingly, this independently determined result is consistent with the outcome of the experimental approach.

\section{Conclusions}

Two independent approaches were undertaken to compare the different models, one being an experimental approach and the other using finite element modelling. In the case of the experimental approach, the difficulties in carrying out accurate compression tests have been highlighted. The major issues are correcting for instrument compliance and setting up the instrument with platens that are parallel to each other and at right angles to the applied force. These issues were addressed by using a video extensometer and keeping the instrument setup constant for all measurements.

PMMA cylinders were machined into smaller diameter cylinders as well as cuboid shapes, and both cylinders and cuboids were compressed both axially and transversely. Machining of the initial cylinders to a smaller diameter showed that machining resulted in an increase in the transverse modulus of $4 \%$ and an increase in longitudinal modulus of $7 \%$. These increases were ascribed to a release of stress in the cylinders upon machining. The release of stress was confirmed by viewing both the initial and the machined samples under crossed polars.

Transverse compression of a new set of cylinders yielded force-compression curves after which the cylinders were machined into cuboids and tested. The data from the transverse compression of the cuboids were used to calculate a transverse modulus of $2867 \pm 164 \mathrm{MPa}$ for the machined PMMA. Data from the axial compression of the cuboids were used to calculate a Poisson's ratio of 0.399 .

The transverse force-compression curves of the machined cylinders were analysed using several different theoretical models which allow the calculation of the transverse modulus of fibres/cylinders from compression experiments. These values were compared to the value obtained from the transverse compression of the cuboids. The model developed by Morris was shown to best fit the experimental transverse compression data giving a corrected transverse modulus of $2888 \mathrm{MPa}$ which was $1 \%$ higher, but statistically identical $(p=0.654)$ to the value obtained by direct compression of the cuboid. The Jawad and Ward model, as well as the mathematically equivalent models derived by Phoenix et al. and Lundberg, gave results that were within $8 \%$ of the value obtained from the transverse compression of the cuboids although these results were no longer statistically identical $(p=0.031)$.

A separate and independent approach using finite element numerical modelling was adopted, and the results were compared with two of the analytical models. The finite element approach gave results that lie between the Jawad and Ward and Morris models. The close agreement in the outcomes of the finite element modelling and the experimental approach is indeed most encouraging. Considering the results from both approaches, and given the difficulties of carrying out compression tests and the uncertainties involved in several of the measurements, we conclude that the most accurate of the different analytical models are the equations by Morris as well as those due to Jawad and Ward, Phoenix et al. and Lundberg.

Acknowledgements We would like to acknowledge CSIRO for funding this work and Niall Finn for critical reading of the manuscript and helpful suggestions.

\section{Compliance with ethical standards}

Conflict of interest The authors declare that they have no conflict of interest.

\section{References}

1. Cheng M, Chen WM, Weerasooriya T (2004) Experimental investigation of the transverse mechanical properties of a single Kevlar KM2 fiber. Int J Solids Struct 41(22-23):6215-6232

2. Kawabata S (1990) Measurement of the transverse mechanical properties of high-performance fibers. J Text Inst 81 (4):432-447

3. Lim J et al (2010) Mechanical behavior of A265 single fibers. J Mater Sci 45(3):652-661

4. Morton WE, Hearle JWS (1993) Physical properties of textile fibres, 3rd edn. The Textile Institute, Manchester 
5. Pinnock PR, Ward IM (1963) Dynamic mechanical measurements on polyethylene terephthalate. Proc Phys Soc 81(2):260-275

6. Coleman JN et al (2006) Small but strong: a review of the mechanical properties of carbon nanotube-polymer composites. Carbon 44:1624-1652

7. Mittal V (ed) (2013) Modeling and prediction of polymer nanocomposite properties. Wiley, New York

8. Tan P, Tong L, Steven GP (1997) Modelling for predicting the mechanical properties of textile composites-a review. Compos A 28A:903-922

9. Torquato $\mathrm{S}$ (2000) Modeling of physical properties of composite materials. Int J Solids Struct 37:411-422

10. Hadley DW, Ward IM, Ward J (1965) The transverse compression of anisotropic fibre monofilaments. Proc R Soc Lond Ser A 285:275-286

11. Fujita K, Sawada Y, Nakanishi Y (2001) Effect of cross-sectional textures on transverse compressive properties of pitch-based carbon fibers. J Soc Mater Sci Jpn 7(2):116-121

12. Naito K, Tanaka Y, Yang J-M (2017) Transverse compressive properties of polyacrylonitrile (PAN)-based and pitch-based single carbon fibers. Carbon 118:168-183

13. Phoenix SL, Skelton J (1974) Transverse compressive moduli and yield behavior of some orthotropic, high-modulus filaments. Text Res J 44(12):934-940

14. Jones MCG et al (1997) The lateral deformation of cross-linkable PPXTA fibres. J Mater Sci 32(11):2855-2871

15. Singletary J et al (2000) The transverse compression of PPTA fibers - part I-single fibre transverse compression testing. J Mater Sci 35(3):583-592

16. Sockalingam $S$ et al (2016) Transverse compression behavior of Kevlar KM2 single fiber. Compos A Appl Sci Manuf 81:271-281

17. Hadley DW, Pinnock PR, Ward IM (1969) Anisotropy in oriented fibres from synthetic polymers. J Mater Sci 4(2):152-165

18. Kotani T, Sweeney J, Ward IM (1994) The measurement of transverse mechanical properties of polymer fibres. J Mater Sci 29(21):5551-5558

19. Pinnock PR, Ward IM, Wolfe JM (1966) The compression of anisotropic fibre monofilaments. II. Proc R Soc Lond A 291(1425):267-278

20. Stamoulis G, Wagner-Kocher C, Renner M (2007) Experimental study of the transverse mechanical properties of polyamide 6.6 monofilaments. J Mater Sci 42(12):4441-4450

21. M'Ewen E, Li X (1949) Stresses in elastic cylinders in contact along a generatrix (including the effect of tangential friction). Philos Mag Ser 40(7):454-459

22. Morris $S(1968)$ The determination of the lateral-compression modulus of fibres. J Text Inst 59(11):536-547

23. Jawad SA, Ward IM (1978) The transverse compression of oriented nylon and polyethylene extrudates. J Mater Sci 13(7):1381-1387

24. Foppl A (1907) Die wichtigsten lehren der hoheren elastizitatstheorie, in Vorlesungen uber Technische Mechanik. B. G. Teubner, Leipzig, pp 311-372
25. Lundberg G (1949) Cylinder compressed between two plane bodies. As cited by H. McCallion and N. Truong, The deformation of rough cylinders compressed between smooth flat surfaces of hard blocks. Wear 79(3):347-361

26. Sherif SM, Segerlind LJ, Frame JS (1976) An equation for the modulus of elasticity of a radially compressed cylinder. Trans Am Soc Agric Eng 19(4):782-791

27. Kar NK et al (2012) Diametral compression of pultruded composite rods. Compos Sci Technol 72:1283-1290

28. Hillbrick $L$ et al (2013) Transverse modulus of carbon fibres. Proceedings of the fiber society spring meeting and technical conference. In: The fiber society spring meeting and technical conference, Geelong, Australia

29. Hillbrick L et al (2015) Determination of the transverse modulus of cylindrical samples by compression between two parallel flat plates, in Carbon fibre-future directions conference, Geelong, Australia

30. Hertz $\mathrm{H}$ (1896) On the contact of elastic solids. Miscellaneous papers, chap 5. MacMillan, London

31. Hertz $\mathrm{H}$ (1896) On the contact of rigid elastic solids and on hardness. Miscellaneous papers, chap 6. MacMillan, London

32. McCallion $\mathrm{H}$, Truong $\mathrm{N}$ (1982) The deformation of rough cylinders compressed between smooth flat surfaces of hard blocks. Wear 79(2):347-361

33. Zantopulos $H$ (1988) An alternate solution of the deformation of a cylinder between two flat plates. J Tribol 110:727-729

34. Hoeprich MR, Zantopulos H (1981) Line contact deformation: a cylinder between two flat plates. J Tribol 103(1):21-25

35. Kosarev OI (2010) Contact deformation of a cylinder under its compression by two flat plates. J Mach Manuf Reliab 39(4):359-366

36. David OB et al (2013) Evaluation of the mechanical properties of PMMA reinforced with carbon nanotubes-experiments and modeling. Exp Mech 54:175-186

37. Musgrave M (1990) On the constraints of positive-definite strain energy in anisotropic elastic media. Q J Mech Appl Mech 43:605-621

38. Kese KO, Li ZC, Bergman B (2004) Influence of residual stress on elastic modulus and hardness of soda-lime glass measured by nanoindentation. J Mater Res 19(10):3109-3119

39. Dörr VJ (1955) Oberflachenverformungen und Randkrafte bei runden Rollen und Bohrungen. Der Stahlbau 24:202-206

40. Mott PH, Roland CM (2009) Limits to Poisson's ratio in isotropic materials. Phys Rev B 80:132104

41. Mott PH, Roland CM (2013) Limits to Poisson's ratio in isotropic materials. General result for arbitrary deformation. Phys Scr 87(5):055404

Publisher's Note Springer Nature remains neutral with regard to jurisdictional claims in published maps and institutional affiliations. 